\title{
Multi-dot floating-gates for nonvolatile semiconductor memories: Their ion beam synthesis and morphology
}

T. Müller, K.-H. Heinig, W. Möller, C. Bonafos, H. Coffin, N. Cherkashin, G. Ben Assayag, S. Schamm, G. Zanchi, A. Claverie, M. Tencé, and C. Colliex

Citation: Appl. Phys. Lett. 85, 2373 (2004); doi: 10.1063/1.1794856

View online: https://doi.org/10.1063/1.1794856

View Table of Contents: http://aip.scitation.org/toc/apl/85/12

Published by the American Institute of Physics

\section{Articles you may be interested in}

Size and location control of Si nanocrystals at ion beam synthesis in thin $\mathrm{SiO}_{2}$ films

Applied Physics Letters 81, 3049 (2002); 10.1063/1.1512952

Sponge-like $\mathrm{Si}_{-} \mathrm{SiO}_{2}$ nanocomposite-Morphology studies of spinodally decomposed silicon-rich oxide Applied Physics Letters 103, 131911 (2013); 10.1063/1.4820453

Formation and coarsening of sponge-like $\mathrm{Si}_{-} \mathrm{SiO}_{2}$ nanocomposites

Applied Physics Letters 103, 133106 (2013); 10.1063/1.4822125

Manipulation of two-dimensional arrays of $\mathrm{Si}$ nanocrystals embedded in thin $\mathrm{SiO}_{2}$ layers by low energy ion implantation

Journal of Applied Physics 95, 5696 (2004); 10.1063/1.1695594

Stress measurements of germanium nanocrystals embedded in silicon oxide

Journal of Applied Physics 94, 5639 (2003); 10.1063/1.1617361

Layer morphology and Al implant profiles after annealing of supersaturated, single-crystalline, amorphous, and nanocrystalline SiC

Journal of Applied Physics 96, 2841 (2004); 10.1063/1.1769600

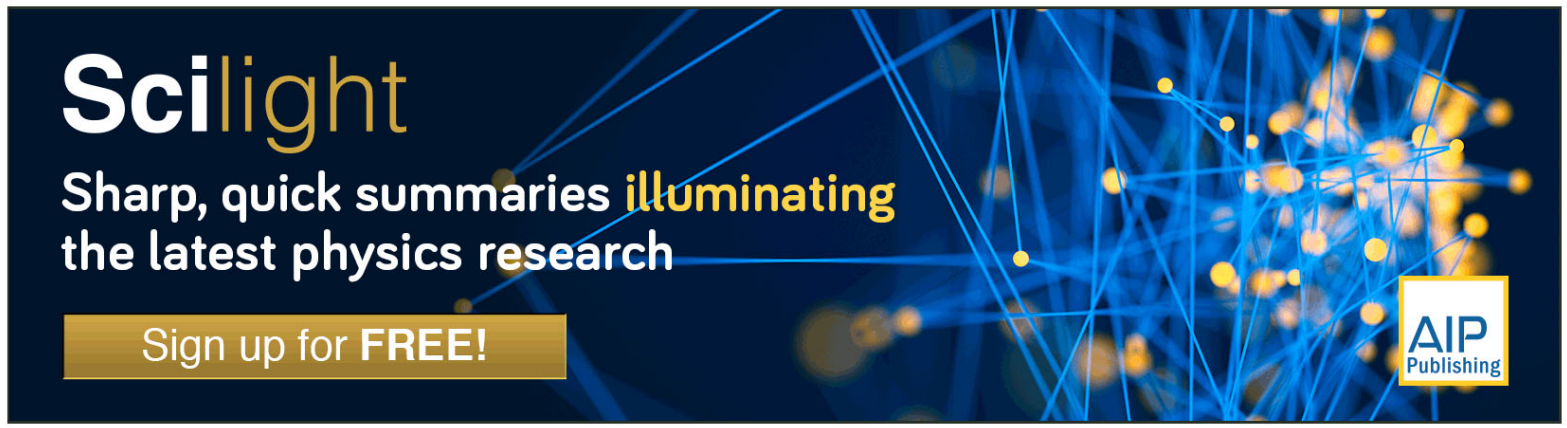




\title{
Multi-dot floating-gates for nonvolatile semiconductor memories: Their ion beam synthesis and morphology
}

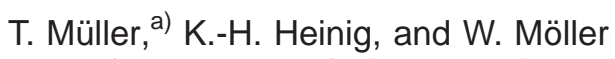 \\ Research Center Rossendorf, Institute of Ion Beam Physics and Materials Research, P.O. Box 5101 19, \\ 01314 Dresden, Germany \\ C. Bonafos, H. Coffin, N. Cherkashin, ${ }^{\text {b) }}$ G. Ben Assayag, S. Schamm, \\ G. Zanchi, and A. Claverie \\ nMat Group, CNRS/CEMES, 29 Rue Jeanne Marvig, 31055 Toulouse, France \\ M. Tencé and C. Colliex \\ Laboratoire de Physique des Solides, Université Paris-Sud-UMR 8502, 91405 Orsay, France
}

(Received 9 February 2004; accepted 20 July 2004)

\begin{abstract}
Scalability and performance of current flash memories can be improved substantially by replacing the floating polycrystalline-silicon gate by a layer of Si dots. Here, we present both experimental and theoretical studies on ion beam synthesis of multi-dot layers consisting of Si nanocrystals (NCs) embedded in the gate oxide. Former studies have suffered from the weak $Z$ contrast between $\mathrm{Si}$ and $\mathrm{SiO}_{2}$ in transmission electron microscopy (TEM). This letter maps $\mathrm{Si}$ plasmon losses with a scanning TEM equipped with a parallel electron energy loss spectroscopy system. Kinetic Monte Carlo simulations of Si phase separation have been performed and compared with Si plasmon maps. Predicted and measured Si morphologies agree remarkably well, both change with increasing ion fluence from isolated NCs to spinodal pattern. However, the predicted fluences are lower than the experimental ones. We identify as the main reason of this discrepancy the partial oxidation of implanted $\mathrm{Si}$ by atmospheric humidity, which penetrates into the as-implanted $\mathrm{SiO}_{2}$. (C) 2004 American Institute of Physics. [DOI: 10.1063/1.1794856]
\end{abstract}

Metal-oxide-silicon field-effect transistors with an electrically isolated ("floating") gate layer embedded in the gate oxide are currently used as flash memories. The replacement of this floating-gate by a layer of discrete Si nanocrystals $(\mathrm{NCs})^{1}$ improves the performance of flash memories substantially. ${ }^{2}$ The reduced probability for a complete discharging of the multi-dot floating-gate by oxide defects allows thinner tunnel oxides. In turn, the floating-gate will be charged/discharged by quantum mechanical direct electron tunneling (instead of defect-generating Fowler-Nordheim tunneling). The memory operation voltage can be reduced and scalability is improved. Using ion beam synthesis, the multi-dot floating-gate can be fabricated along with standard (CMOS) processing. ${ }^{3} \mathrm{Si}^{+}$ions are implanted at ultralow energies into the gate oxide, causing there a high Si supersaturation. During postimplantation annealing, this Si supersaturation leads to phase separation of elemental $\mathrm{Si}$ from $\mathrm{SiO}_{2}{ }^{4}$ Imaging this phase separation process is difficult. Transmission electron microscopy (TEM) has historically suffered from weak $Z$ contrast between $\mathrm{Si}$ and $\mathrm{SiO}_{2}$ phases. Recently, this problem was partially overcome by Fresnel imaging using under-focused bright field conditions. ${ }^{5}$ Thus, the distance of the layer of phase separated Si from the transistor channel could be determined. ${ }^{6,7}$ However, this technique fails to resolve the morphology of the phase separated Si. For instance, recent kinetic Monte Carlo (KMC) simulations of phase separation predict a pronounced fluence dependence of the precipitate morphology. ${ }^{4}$ For low $\mathrm{Si}^{+}$fluences, spherical and isolated Si NCs form by nucleation and growth, while for

\footnotetext{
a)Electronic mail: t.mueller@fz-rossendorf.de

${ }^{b}$ On leave from: Ioffe Physico-Technical Institute, St. Petersburg.
}

higher $\mathrm{Si}^{+}$fluences spinodal decomposition occurs. The elongated, non-spherical Si structures, formed by spinodal decomposition, coalesce at even higher fluences to an interconnected, labyrinthine Si network. This letter uses a scanning TEM (STEM) with an efficient parallel electron energy loss spectroscopy (PEELS) system, to confirm the predictions of the KMC simulations. The contrast problem of conventional TEM could be overcome by mapping Si plasmon losses, which differ from $\mathrm{SiO}_{2}$. Comprehensive $\mathrm{KMC}$ studies and PEELS-STEM analysis has been performed to understand the complex process of phase separation in a thin buried layer.

To form $\mathrm{Si} \mathrm{NCs}$ by phase separation, $\mathrm{Si}^{+}$ions have been implanted at $1 \mathrm{keV}$ energy into 10 -nm-thick $\mathrm{SiO}_{2}$ layers, which were thermally grown on (001) Si substrates. Using an AXCELIS GSD-ULTRA ultralow-energy implanter, fluences of $5 \times 10^{15}, 1 \times 10^{16}$, and $2 \times 10^{16} \mathrm{~cm}^{-2}$ were implanted at room temperature. Surface charging due to implantation was compensated by a Xe plasma electron flood gun. The implanted samples were cleaned using a piranha solution and furnace annealed for $30 \mathrm{~min}$ in $\mathrm{N}_{2}$ at $950{ }^{\circ} \mathrm{C}$. From all samples, cross sectional and plane view TEM specimens were prepared by the standard procedure of grinding, dimpling, and $\mathrm{Ar}^{+}$ion beam thinning. PEELS-STEM was performed on plane view samples using a scanning TEM VG-HB 501 operating at $100 \mathrm{kV}$ that is equipped with a field emission cathode and a parallel Gatan 666 EELS spectrometer. Low-loss EELS spectra were recorded at each picture point, hence a spectrum image was acquired. ${ }^{8}$ These spectra were fitted in the energy range of $15-30 \mathrm{eV}$ by a weighted sum of two plasmon reference spectra, which were obtained from bulk $\mathrm{Si}$ and $\mathrm{SiO}_{2}$. Potential size effects like shifts of the $\mathrm{Si}$ plasmon resonance of small Si NCs were not compen- 

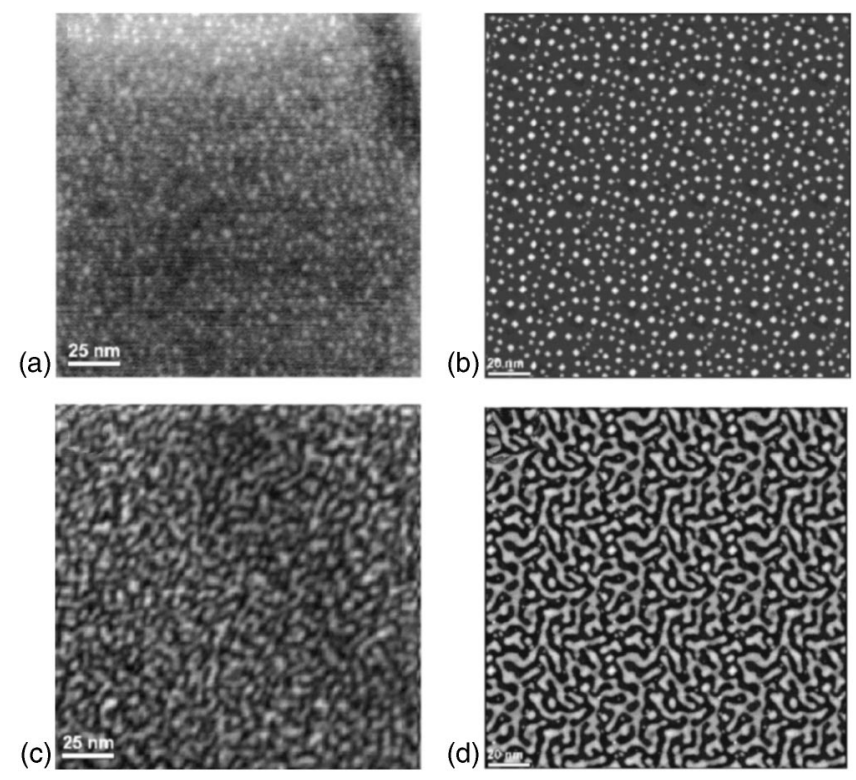

FIG. 1. Morphology of phase separated $\mathrm{Si}$ in $\mathrm{SiO}_{2}$. Si plasmon maps by PEELS-STEM (a),(c) are compared to corresponding KMC simulations (b),(d). Nucleation of $\mathrm{Si} \mathrm{NCs}$ is observed (a) for a Si fluence of 1 $\times 10^{16} \mathrm{~cm}^{-2}$ and predicted with the same morphology for (b) $3 \times 10^{15} \mathrm{~cm}^{-2}$. Spinodal patterns are imaged (c) for $2 \times 10^{16} \mathrm{~cm}^{-2}$ and simulated (d) for 8 $\times 10^{15} \mathrm{~cm}^{-2}$. Bright and dark regions correspond to $\mathrm{Si}$ and $\mathrm{SiO}_{2}$ phases, respectively.

sated. The gray level of the PEELS-STEM image pixels is given by the ratio of the intensity of the Si plasmon peak and the total intensity of the $\mathrm{Si}$ and $\mathrm{SiO}_{2}$ plasmon peaks and, therefore, maps the Si bulk plasmons. Quantitative Si concentrations, however, cannot be given, only the relative $\mathrm{Si}$ content is imaged. In Fig. 1, such Si plasmon maps (on the left-hand side) are compared to plane view snapshots of KMC simulations (on the right-hand side). This atomistic approach to phase separation of excess $\mathrm{Si}$ in thin $\mathrm{SiO}_{2}$ layers by atomistic simulations was recently described in detail in Refs. 4 and 9. The depth profiles of excess $\mathrm{Si}$ due to high fluence $\mathrm{Si}^{+}$ion implantation into thin gate oxides have been calculated by the binary collision program TRIDYN ${ }^{10}$ accounting for the effects of ion erosion, target swelling, and ion beam mixing dynamically. The postimplantation phase separation during thermal treatment is described by a kinetic three-dimensional lattice Monte Carlo program package. ${ }^{11,12}$ Taking the TRIDYN profiles of Si excess as well as Si solubility and diffusivity in $\mathrm{SiO}_{2}$ as input, the program describes excess $\mathrm{Si}$ diffusion, precipitation, and Ostwald ripening in the thin $\mathrm{SiO}_{2}$ layer under the constraints of the boundary conditions of a nearby $\mathrm{Si} / \mathrm{SiO}_{2}$ interface and a free $\mathrm{SiO}_{2}$ surface. Here, we use a simplified version of the KMC program, i.e., only Ising-type nearest-neighbor interactions. It should be noted that KMC simulations with measured $\mathrm{Si}$ self-diffusivities ${ }^{13,14}$ lead to too long annealing times or too high temperatures. Obviously, the diffusive Si mass transport by a mobile $\mathrm{SiO}_{2}$ defect with local $\mathrm{Si}$ excess (that could either be named $\mathrm{Si}$ interstitial, $\mathrm{SiO}$ molecule, ${ }^{13}$ or oxygen vacancy ${ }^{15}$ ) does not necessarily follow the same mechanism as the ${ }^{28} \mathrm{SiO}_{2} /{ }^{30} \mathrm{SiO}_{2}$ interface broadening, which was analyzed in self-diffusivity studies. In this letter, the discrepancy between diffusive Si mass transport and $\mathrm{Si}$ self-diffusivity will not be discussed as our theoretical predictions aim at the reaction pathway of $\mathrm{SiO}_{x}$ decomposition rather than at a quantitative prediction of annealing time and temperature.
Thus, KMC simulations utilize a relative time scale "Monte Carlo steps" (MCS) that allows a posterior recalibration with realistic Si diffusivities. ${ }^{12}$

Image processing of $\mathrm{KMC}$ simulation data allowed one to obtain KMC simulation snapshots that can be compared directly to PEELS-STEM or Fresnel images. In plane view, the simulation cell has been tripled laterally taking advantage of the periodic boundary conditions. The number of excess $\mathrm{Si}$ atoms in the vertical column below the pixel at $(x, y)$ determines its gray level. The highest occurring number of $\mathrm{Si}$ atoms in such a column is assigned to white, whereas black means that no $\mathrm{Si}$ excess is found in the $\mathrm{SiO}_{2}$ matrix.

No Si precipitates were observed in the sample for the lowest $\mathrm{Si}^{+}$fluence of $5 \times 10^{15} \mathrm{~cm}^{-2}$ (TEM images not shown here). For the medium $\mathrm{Si}^{+}$fluence of $1 \times 10^{16} \mathrm{~cm}^{-2}$, white spots in a dark background appear in the plane view Si plasmon map shown in Fig. 1(a) indicating spherical Si NCs embedded in the $\mathrm{SiO}_{2}$ matrix. During thermal annealing, these NCs have formed by nucleation and growth in the $\mathrm{Si}$ enriched region of the implanted $\mathrm{SiO}_{2}$ layer. The observed NCs are relatively small with an estimated mean diameter of $2.7 \mathrm{~nm}$ and have a high area density of $3.3 \times 10^{12} \mathrm{~cm}^{-2}$. A similar precipitate morphology with the same mean NC size and density is found in the KMC simulation snapshot of Fig. 1(b), but for a significantly lower $\mathrm{Si}^{+}$fluence of 3 $\times 10^{15} \mathrm{~cm}^{-2}$ and a KMC simulation (annealing) time of 2100 kMCS. It should be noted that even this "medium" experimental $\mathrm{Si}^{+}$fluence of $1 \times 10^{16} \mathrm{~cm}^{-2}$ leads in the KMC simulation to the formation of coalesced poly-Si layer buried in the $\mathrm{SiO}_{2}{ }^{4}$ For the highest $\mathrm{Si}^{+}$fluence of $2 \times 10^{16} \mathrm{Si}^{+} \mathrm{cm}^{-2}$, the morphology of the phase separated Si has changed completely as observed in Fig. 1(c). A spaghetti-like network of white and black regions is found. This pattern clearly indicates that phase separation took place by spinodal decomposition. Even more, percolation is observed. Si precipitates are no longer spatially isolated, but an interconnected network of phase separated Si has formed. An equivalent morphology with the same typical distances as in Fig. 1(c) between the spinodal fingers is found in the KMC simulation snapshot of Fig. 1(d). This result was obtained for $8 \times 10^{15} \mathrm{Si}^{+} \mathrm{cm}^{-2}$ and a simulation time of $300 \mathrm{kMCS}$. Strikingly, the morphology agrees remarkably well between both images, while simulation snapshots for other fluences or annealing times deviate instead considerably from the morphology seen in Fig. 1(c).

Samples were studied additionally in cross section using a CM30 Phillips TEM equipped with a $\mathrm{LaB}_{6}$ cathode operating at $300 \mathrm{kV}$. Fresnel imaging conditions were applied in order to achieve at least a weak contrast between $\mathrm{Si}$ and $\mathrm{SiO}_{2}$ in cross sectional TEM (XTEM) images, ${ }^{5}$ i.e., images were taken at out-of-Bragg alignment and under strongly underfocused bright field conditions. Details of the responsible contrast mechanism are given in Ref. 5. Figures 2(a) and 2(c) show the XTEM Fresnel images for fluences of 1 $\times 10^{16} \mathrm{Si}^{+} \mathrm{cm}^{-2}$ and $2 \times 10^{16} \mathrm{Si}^{+} \mathrm{cm}^{-2}$, respectively. Cross section KMC simulation snapshots are displayed in Figs. 2(b) and 2(d) for $3 \times 10^{15} \mathrm{Si}^{+} \mathrm{cm}^{-2}$ (2100 kMCS) and 8 $\times 10^{15} \mathrm{Si}^{+} \mathrm{cm}^{-2}$ (300 kMCS), respectively. Thereby, plane view image processing was applied to KMC cross section views with an inverted gray scale. In all images, the phase separated $\mathrm{Si}$ forms a single, sharp layer seen as gray band in the $\mathrm{SiO}_{2}$ that is well separated from the $\mathrm{SiO}_{2} / \mathrm{Si}$ interface. Due to the extremely shallow $\mathrm{Si}$ excess profile obtained by low-energy $\mathrm{Si}^{+}$implantation, phase separation is quasicon- 

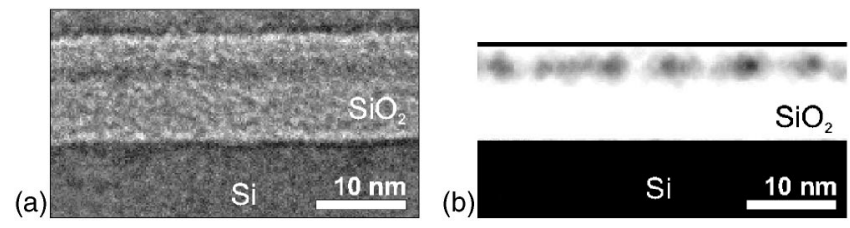

(c)
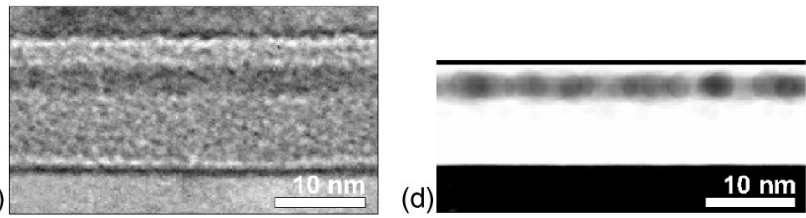

FIG. 2. Cross-section view of the layer of phase separated $\mathrm{Si}$ in $\mathrm{SiO}_{2}$. Fresnel XTEM images for (a) $1 \times 10^{16} \mathrm{Si}^{+} \mathrm{cm}^{-2}$ and (c) $2 \times 10^{16} \mathrm{Si}^{+} \mathrm{cm}^{-2}$ are compared to cross-sectional KMC simulation snapshots for (b) 3 $\times 10^{15} \mathrm{Si}^{+} \mathrm{cm}^{-2}$ and (d) $8 \times 10^{15} \mathrm{Si}^{+} \mathrm{cm}^{-2}$.

fined to two dimensions. For $1 \times 10^{16} \mathrm{Si}^{+} \mathrm{cm}^{-2}$, Si precipitates align nicely in a thin layer, which is just a few nanometers thick, Fig. 2(a). When the $\mathrm{Si}^{+}$fluences are increased to $2 \times 10^{16} \mathrm{Si}^{+} \mathrm{cm}^{-2}$, the precipitate layer remains comparatively well localized in depth, Fig. 2(c), although the Si morphology observed in plane view has changed completely, Fig. 1(c). The total $\mathrm{SiO}_{2}$ thickness is systematically smaller for the KMC simulations than for the corresponding Fresnel XTEM images. Nevertheless, the distance between the $\mathrm{Si} / \mathrm{SiO}_{2}$ interface and the phase separated $\mathrm{Si}$ agrees nicely for experiment and simulation.

The Si fluences in our KMC simulations were chosen in order to obtain morphologies of phase separated $\mathrm{Si}$, which are similar to experimental ones. This adjustments of our simulation reveals a strong discrepancy between experimental and theoretical Si fluences. More Si than theoretically predicted has to be implanted. Not all Si that has nominally been implanted into the $\mathrm{SiO}_{2}$ is available for phase separation. The reason for the missing Si excess might be twofold. (i) Recent time of flight secondary ion mass spectroscopy measurements on low-energy, low-fluence ${ }^{30} \mathrm{Si}^{+}$as-implanted $\mathrm{SiO}_{2}$ samples indicate that only a fraction of about $0.5-0.7$ of the nominal $\mathrm{Si}^{+}$fluence has been implanted into the $\mathrm{SiO}_{2}{ }^{16}$ (ii) It is known that ( $\mathrm{Si}$ or $\mathrm{Ge}$ ) NC formation in very thin $\mathrm{SiO}_{2}$ films is extremely sensitive to humidity absorbed by the as-implanted (damaged) glass network ${ }^{17}$ as well as to oxidants being present in the annealing ambient. ${ }^{18}$ Thus, a considerable amount of the implanted Si might become oxidized during annealing. This explains also why no NCs have been observed by TEM for the lowest $\mathrm{Si}^{+}$fluence of 5 $\times 10^{15} \mathrm{~cm}^{-2}$. The implanted Si has been oxidized completely during the thermal annealing. At the same time, oxidation of the implanted $\mathrm{Si}$ leads to a volume expansion that increases the overall $\mathrm{SiO}_{2}$ layer thickness. ${ }^{6}$ This swelling of the $\mathrm{SiO}_{2}$ due to $\mathrm{Si}$ oxidation can be seen in the XTEM images of Figs. 2(a)-2(c) if compared to the KMC simulation snapshots, Figs. 2(b) and 2(d), which just include the $\mathrm{SiO}_{2}$ expansion due to the incorporated Si atoms. ${ }^{9}$ Here, the KMC simulations do not account for oxidation and the swelling caused by it. To do so, a multicomponent $\mathrm{KMC}$ approach $(\mathrm{Si}+\mathrm{O})$ is needed. Though samples have been annealed under fixed ex- perimental conditions, the KMC simulation snapshots of corresponding $\mathrm{Si}$ patterns refer to different simulation times. Two reasons might be responsible for this discrepancy in the evolution speed. At first, the oxidation of a substantial part of implanted Si might influence the kinetics, and second, the $\mathrm{Si}$ bulk diffusion in $\mathrm{SiO}_{2}$ might differ substantially from the $\mathrm{Si}$ diffusion at the $\mathrm{Si} / \mathrm{SiO}_{2}$ interface. Studies that investigate this point are under way.

Summarizing, extensive studies on low-energy ion beam synthesis of multi-dot $\mathrm{Si}$ floating-gates embedded in thin $\mathrm{SiO}_{2}$ layers have been presented. The morphology of this floating-gate layer is imaged by PEELS-STEM. A direct comparison to kinetic three-dimensional lattice Monte Carlo simulation snapshots have been made and show a remarkable agreement between the atomistic simulations and the PEELS-STEM images. A strong fluence dependence of the precipitate morphology is confirmed. For low $\mathrm{Si}^{+}$fluences, isolated Si NCs form by nucleation and growth, while high fluences lead to spinodal pattern. Predicted and measured morphologies agree remarkably well. However, the predicted fluences are lower than the experimental ones. A substantial fraction of the implanted $\mathrm{Si}$ is lost by parasitic oxidation with atmospheric humidity.

This work was supported by the European Commission through the Growth project G5RD/2000/00320—NEON.

${ }^{1}$ S. Tiwari, F. Rana, H. Hanafi, A. Hartstein, E. F. Crabbe, and K. Chan, Appl. Phys. Lett. 68, 1377 (1996).

${ }^{2}$ S. Tiwari, J. A. Wahl, H. Silva, F. Rana, and J. J. Welser, Appl. Phys. A: Mater. Sci. Process. 71, 403 (2000).

${ }^{3}$ E. Kapetanakis, P. Normand, D. Tsoukalas, and K. Beltsios, Appl. Phys. Lett. 80, 2794 (2002).

${ }^{4}$ T. Müller, K.-H. Heinig, and W. Möller, Appl. Phys. Lett. 81, 3049 (2002).

${ }^{5}$ G. B. Assayag, C. Bonafos, M. Carrada, P. Normand, D. Tsoukalas, and A. Claverie, Appl. Phys. Lett. 82, 200 (2003).

${ }^{6}$ M. Carrada, N. Cherkashin, C. Bonafos, G. Benassayag, D. Chassaing, P. Normand, D. Tsoukalas, V. Soncini, and A. Claverie, Mater. Sci. Eng., B 101, 204 (2003).

${ }^{7}$ C. Bonafos, M. Carrada, N. Cherkashin, H. Coffin, D. Chassaing, G. Ben Assayag, A. Claverie, T. Müller, K. H. Heinig, M. Perego, M. Fanciulli, P. Normand, and D. Tsoukalas, J. Appl. Phys. 95, 5696 (2004).

${ }^{8}$ C. Jeanguillaume and C. Colliex, Ultramicroscopy 28, 252 (1989).

${ }^{9}$ T. Müller, K.-H. Heinig, and W. Möller, Mater. Sci. Eng., B 101/1-3, 49 (2003).

${ }^{10}$ W. Möller, and W. Eckstein, Nucl. Instrum. Methods Phys. Res. B 2, 814 (1984).

${ }^{11}$ K.-H. Heinig, T. Müller, B. Schmidt, M. Strobel, and W. Möller, Appl. Phys. A: Mater. Sci. Process. 77, 17 (2003).

${ }^{12}$ M. Strobel, K.-H. Heinig, and W. Möller, Phys. Rev. B 64, 245422 (2001).

${ }^{13}$ M. Uematsu, H. Kageshima, Y. Takahashi, S. Fukatsu, K. M. Itoh, K. Shiraishi, and U. Gösele, Appl. Phys. Lett. 84, 876 (2004).

${ }^{14}$ D. Mathiot, J. P. Schunck, M. Perego, M. Fanciulli, P. Normand, C. Tsamis, and D. Tsoukalas, J. Appl. Phys. 94, 2136 (2003).

${ }^{15}$ J. Song, L. R. Corrales, G. Kresse, and H. Jonsson, Phys. Rev. B 64, 134102 (2001).

${ }^{16}$ M. Perego, M. Fanculli, G. Ben Assayag, and A. Claverie, (private communication).

${ }^{17}$ B. Schmidt, D. Grambole, and F. Herrmann, Nucl. Instrum. Methods Phys. Res. B 191, 482 (2002).

${ }^{18}$ S. Oswald, B. Schmidt, and K.-H. Heinig, Surf. Interface Anal. 29, 249 (2000). 